\title{
Implante de testosterona para la mujer, evaluación de seguridad y efectividad en esta vía de administración
}

\section{Testosterone implant for women, evaluation of safety and effectiveness in this route of administration}

\author{
Margot A. Acuña-San Martín* y René P. Gallardo-González \\ Departamento Obstetricia y Ginecología, Facultad de Medicina, Universidad de La Frontera, Temuco, Chile
}

\section{Resumen}

Introducción y objetivo: El rol de la testosterona exógena en la función sexual femenina ha sido estudiado durante muchos años, con resultados contradictorios. En el último tiempo se ha promovido el uso de pellets de testosterona como una solución para mejorar la libido femenina, la cognición, la fuerza muscular y los sistemas cardiovascular y óseo, e incluso evitar el envejecimiento. Por ello, revisamos las publicaciones para tratar de responder si esto es una moda o el tratamiento más innovador del último tiempo. Método: Se analizaron las bases de datos PubMed/Medline, Trip Database, Cochrane, SciELO, Scopus, UpToDate, Ovid, ProQuest, Science Direct y ResearchGate. Resultados: De acuerdo con la evidencia, la mejor testosterona disponible es la transdérmica y debe ser usada solo en el trastorno del deseo sexual hipoactivo (TDSH). Los trabajos que evalúan los pellets de testosterona tienen sesgos metodológicos importantes. Si bien son útiles para mejorar la función sexual femenina, producen concentraciones plasmáticas suprafisiológicas de testosterona, por lo que no se puede establecer su seguridad a largo plazo. Tampoco hay datos suficientes que avalen su uso para mejorar el rendimiento cognitivo y el bienestar general, en el tratamiento de enfermedades cardiovasculares o en la prevención de enfermedad ósea. Conclusiones: La testosterona solo se recomienda en el tratamiento del TDSH por vía transdérmica. No recomendamos el uso de pellets de testosterona para el tratamiento de la disfunción sexual ni como hormona antienvejecimiento, ya que no hay estudios consistentes sobre su seguridad, eficacia y efectos adversos a largo plazo.

Palabras clave: Biosíntesis de andrógenos. Testosterona. Implantes de testosterona. Chip de testosterona. Pellet de testosterona. Trastorno del deseo sexual hipoactivo.

\section{Abstract}

Introduction and objective: The role of exogenous testosterone in female sexual function has been studied for many years with contradictory results. In recent times, the use of testosterone pellets has been promoted as a solution to improve female libido, cognition, muscle strength, cardiovascular system, bone and even prevent aging. Therefore, we will review the publications in order to answer whether this is a fad or the most innovative treatment of recent times. Method: The databases PubMed/Medline, Trip Database, Cochrane, SciELO, Scopus, UpToDate, Ovid, ProQuest, Science Direct and ResearchGate were analyzed. Results: So far, the evidence best testosterone available is transdermal testosterone and that it should be used only in hypoactive sexual desire disorder (HSDD). Papers evaluating testosterone pellets have significant methodological biases. While they are useful in improving female sexual function, they produce supra-physiological plasma levels of testosterone, so their long-term safety cannot be established. There is also insufficient data to support their use in improving

Disponible en internet: 07-02-2022 Rev Chil Obstet Ginecol. 2021;86(6):516-520

www. rechog.com 0048-766X / @ 2021 Sociedad Chilena de Obstetricia y Ginecología. Publicado por Permanyer. Éste es un artículo open access bajo la licencia CC BY-NC-ND (https://creativecommons.org/licenses/by-nc-nd/4.0/). 
cognitive performance and general well-being, treatment of cardiovascular disease or prevention of bone disease. Conclusions: Testosterone is only recommended for the tratment of HSDD via the transdermal route. We do not recommended the use of testosterone pellets for the treatment of sexual dysfunction or as an anti aging hormone, as there are no consistent studies on its safety, efficacy, and long-term adverse effects.

Keywords: Androgen biosynthesis. Testosterone. Testosterone implant. Testosterone chip. Testosterone pellets. Hypoactive sexual desire disorder.

\section{Introducción}

Los andrógenos son esteroides con 19 átomos de carbono, encargados de múltiples funciones en los varones, pero que también cumplen un papel importante en la mujer. Actúan siendo precursores de los estrógenos y mediante interacción directa con aquellos tejidos que poseen receptores afines, los cuales se encuentran ampliamente distribuidos en el cuerpo de la mujer ${ }^{1,2}$.

Durante el último siglo se ha estudiado el potencial terapéutico de los andrógenos en la mujer. Es así como la testosterona se ha utilizado en diferentes formatos para combatir la disfunción sexual femenina, específicamente el trastorno de deseo sexual hipoactivo (TDSH), según la última actualización del DSM-5³.

Se ha preconizado en los últimos años, en Chile y en el resto del mundo, el tratamiento con pellets de testosterona bioidéntica con prometedores beneficios como «hormona del rejuvenecimiento», para mejorar la masa muscular, el ánimo y la libido; para reducir el riesgo de diabetes tipo 2, enfermedades cardiacas y osteoporosis; y para mejorar la función cognitiva, reducir la depresión y la ansiedad, y aumentar los niveles de energía corporal. Cabe entonces preguntarse: ¿es esta terapia una simple moda, un gran negocio o, efectivamente, estamos frente al tratamiento hormonal bioidéntico más innovador del último tiempo?

\section{Método}

Se realizó una búsqueda bibliográfica en las bases de datos más importantes: PubMed/Medline, Trip Database, Cochrane, SciELO, Scopus, UpToDate, Ovid, ProQuest, Science Direct y ResearchGate. Se utilizaron las siguientes palabras clave: biosíntesis de andrógenos, andrógenos, testosterona, implantes de testosterona, chip de testosterona, pellets de testosterona, implantes subcutáneos, testosterona bioidéntica y disfunción sexual femenina. De esta revisión se seleccionaron 24 publicaciones debido a la recurrente mención en diferentes revisiones sistemáticas y guías clínicas, y que cuentan con nivel de evidencia 1 según la guía NICE; además, las últimas publicaciones de sociedades científicas American College of Obstetricians and Gynecologists (ACOG), The International Menopause Society (IMS) y North American Menopause Society (NAMS) en relación con el tema en revisión.

\section{Función de los andrógenos en la mujer}

Los andrógenos en la mujer se producen en las glándulas suprarrenales, el ovario y por conversión hormonal periférica. La testosterona se produce en un 25\% en la glándula suprarrenal, un $25 \%$ en el estroma ovárico y un $50 \%$ por conversión periférica de androstenediona. La producción diaria es de 0,1-0,4 mg, los niveles circulantes durante la vida fertil de la mujer y en la posmenopausia se muestran en la tabla $1^{1}$.

La concentración de testosterona disminuye progresivamente con la edad, por disminución de la producción tanto en la glándula suprarrenal como en el ovario ${ }^{2,4}$.

La testosterona circula en el plasma de tres formas: un $66 \%$ ligada a la globulina fijadora de hormonas sexuales (SHBG), un $33 \%$ ligada a la albúmina y un $1 \%$ libre. Las dos últimas son las formas activas, las cuales pueden tener modificaciones según la cantidad de SHBG circulante. Estas modificaciones pueden traducirse en una disminución o un aumento de la testosterona total, por lo que se recomienda medir la testosterona libre en sangre. El problema es que sus niveles en mujeres son prácticamente indetectables con las técnicas disponibles. Si bien el método de referencia para la medición de la fracción de testosterona libre es la cromatografía líquida con espectrometría de masa, esta se usa solo para fines de investigación y no existe correlación entre las concentraciones plasmáticas y la clínica de las pacientes ${ }^{2,4}$.

La primera relación entre la testosterona y la libido femenina fue descrita por Loeser en 1940 y posteriormente confirmada por Greenblatt en 1942 y por Salmon en 1943. 
Tabla 1. Concentraciones plasmáticas fisiológicas de testosterona total

\begin{tabular}{|l|c|c|}
\hline Edad fértil & Posmenopausia \\
\hline 20 a $75 \mathrm{ng} / \mathrm{dl}$ & 10 a $50 \mathrm{ng} / \mathrm{dl}$ \\
\hline
\end{tabular}

En todo el mundo, al menos 16 millones de mujeres mayores de 50 años experimentan bajo deseo sexual, y aproximadamente 4 millones de ellas presentan un TDSH; por lo tanto, es altamente prevalente ${ }^{5,6}$.

La disminución de la libido aumenta con la edad, siendo que el $26,7 \%$ de todas las mujeres en edad reproductiva y el $54,2 \%$ de todas las mujeres posmenopáusicas la presentan ${ }^{7,8}$.

Los estudios que investigan la correlación del apetito sexual, la excitación sexual y el orgasmo con las mediciones de testosterona total, testosterona libre, androstenediona y deshidroepiandrosterona no han arrojado resultados concluyentes. Esto se debe, entre otras cosas, a que no solo las hormonas desempeñan un papel, sino que también intervienen factores sociales, emocionales y de salud en general ${ }^{1,9}$.

El implante de testosterona fue aprobado por la Food and Drug Administration (FDA) en 1972 para su uso en hombres, pero recién se empezó a comercializar en 2008 con el nombre de Testopel $\AA$, una testosterona cristalina de $75 \mathrm{mg}(3 \times 8 \mathrm{~mm})$, de liberación prolongada, cuyos pellets se insertan en el espacio subcutáneo en la región de la cadera, en un procedimiento ambulatorio, y la dosificación depende del peso del paciente ${ }^{10}$. Llegó como una alternativa a la terapia transdérmica, diferenciándose de ella por no requerir aplicación diaria, tener una alta tasa de cumplimiento y carecer de riesgo de transferencia familiar. Se promocionó como un tratamiento con posible uso en las mujeres y que mantenía unas adecuadas concentraciones séricas de testosterona hasta 6 meses.

A la fecha son escasas las publicaciones en población femenina, y muestran una duración limitada y sesgos metodológicos importantes. De hecho, el uso de testosterona en la mujer no está aprobado por la FDA por falta de estudios que demuestren su seguridad y eficacia a largo plazo ${ }^{2}$.

La mayoría de los estudios se han realizado con pellets de estradiol y testosterona asociados, siendo limitados los reportes con pellets de solo testosterona.

Los estudios a partir de los años 1980, con testosterona sola 0 en asociación con estradiol, dieron resultados contradictorios en cuanto a bienestar general, pero en todos mejoraron la libido, la satisfacción sexual y las fantasías sexuales, a costa de un aumento del hirsutismo y de obtener unos valores plasmáticos de testosterona al menos cuatro veces superiores a los valores normales de las mujeres premenopáusicas, como se muestra en la tabla $2^{11-15}$.

En los años 2009 y 2011, Glaser et al. ${ }^{16,17}$ publicaron datos de los efectos de los pellets de testosterona en el deseo sexual. Utilizando las escalas MRS (Menopause Rating Scale) y de Calidad de Vida Relacionada con la Salud (CVRS), informan que los implantes de testosterona alivian la sintomatología menopáusica, incluyendo el deseo, la actividad y la satisfacción sexual, en mujeres premenopáusicas y posmenopáusicas. Sin embargo, solo informan de las conclusiones, sin los datos estadísticos, y no hacen mención a probables efectos adversos ni utilizan grupo placebo.

En el año 2013, Glaser et al ${ }^{18}$ evaluaron los niveles y los efectos de la testosterona en dosis de $100 \mathrm{mg}$ en pellets, realizando mediciones basales y seriadas de testosterona total. A la cuarta semana, las pacientes tenían valores de cuatro a seis veces sobre el límite normal de producción endógena, independientemente de su índice de masa corporal. Un 85,7\% de las pacientes relataron un aumento de leve a moderado del vello facial, y un $6,4 \%$ un aumento importante. Además, el $11,2 \%$ reportaron un aumento moderado del acné y el $1 \%$ reportó cambios en la voz. Los niveles de testosterona se muestran en la tabla 2.

Braunstein ${ }^{19}$ realizó una revisión de los estudios que tenían metodología adecuada, tanto retrospectivos como prospectivos, publicados hasta el año 2007. Encuentra que la testosterona mejora el TDSH, y recomienda la vía transdérmica por no más allá de 2 años, ya que la seguridad a largo plazo del tratamiento no se puede asegurar al no haber publicaciones de más larga duración. No recomienda la testosterona en pellets por falta de estudios consistentes ${ }^{19,20}$.

En el año 2014, la Endocrine Society (ACOG), American Society for Reproductive Medicine (ASRM), European Society of Endocrinology (ESE) y la IMS publicaron una guía clínica sobre el uso de andrógenos en la mujer. Determinan que se puede usar testosterona en el TDSH, pero no la recomiendan para tratar la infertilidad, el déficit cognitivo, las alteraciones cardiovasculares, las alteraciones metabólicas, la salud ósea ni el bienestar en general, debido a que no hay estudios a largo plazo que demuestren la seguridad de los andrógenos en la mujer. En los estudios analizados encontraron sesgos de metodología, publicación e impresión, e inconsistencias ${ }^{21}$. 
Tabla 2. Concentraciones plasmáticas ( $\mathrm{ng} / \mathrm{dl}$ ) de testosterona con pellets en distintos estudios

\begin{tabular}{|c|c|c|c|c|c|c|c|}
\hline Estudios & 0 meses & 1 mes & 2 meses & 3 meses & 4 meses & 5 meses & 6 meses \\
\hline Kapetanakis, $1982^{* 11}$ & & 250 & & & 20 & & \\
\hline Cardozo, $1984^{* 12}$ & & 77,87 & 63,45 & 77,87 & 72,11 & 109,6 & 74,99 \\
\hline Burger, $1984^{13}$ & 66,3 & 193 & & 121 & & 72 & \\
\hline Burger, $1987^{14}$ & & & & & & & 106,6 \\
\hline Davis, $1995^{* 15}$ & 34,6 & & & & & & 77,8 \\
\hline Glaser, $2013^{18}$ & & 299,3 & & 184,7 & & & \\
\hline
\end{tabular}

Mas recientemente, Islam et al..23, en 2019, realizaron una revisión sistemática de ensayos controlados, aleatorizados y doble ciego, incluyendo todas las vías de administración de testosterona. Incluyeron 36 estudios con un total de 8480 mujeres tratadas con testosterona por al menos 12 semanas entre 1990 y 2018. En todos los estudios encontraron datos incompletos y sesgos de selección. Concluyen que la testosterona, comparada con placebo o estrógeno, mejora significativamente la función sexual (frecuencia satisfactoria de eventos sexuales, deseo sexual, placer, excitación, orgasmo, capacidad de respuesta, reducción de la angustia, imagen y preocupaciones sexuales), pero que faltan datos para asegurar otros beneficios. Los efectos adversos que se reportaron con más frecuencia fueron acné e hirsutismo. Se obtuvieron dosis suprafisiológicas con la administración en pellets e inyectables. Recomiendan el uso de testosterona transdérmica, ya que se mantienen unos valores fisiológicos de testosterona, alrededor de $50 \mathrm{ng} / \mathrm{dl}$, y mencionan que debe haber mayor investigación para evaluar la seguridad a largo plazo; conclusiones a las que había llegado la Cochrane en el año 2010 incluyendo muchos trabajos que se analizan en esta revisión ${ }^{24}$ y que posteriormente Johansen et al. ratifica en el año $2020^{25}$.

En 2019, la revista Climacteric publicó una declaración de consenso global sobre el uso de testosterona en la mujer, en la que participaron las principales sociedades de endocrinología, climaterio y ginecología ${ }^{25}$. Destaca que los efectos adversos más comunes fueron acné y aumento del vello facial y corporal, incluso a dosis fisiológicas. Acepta como único uso de testosterona el TDSH. Advierte que la testosterona bioidéntica no puede ser recomendada para la disfunción sexual, debido a la falta de evidencia sobre su seguridad y eficacia. Por ende, no recomienda el uso de pellets ni de inyecciones, por provocar dosis suprafisiológicas ${ }^{25}$. Además, avala que la vía transdérmica mediante parches de testosterona de $300 \mu \mathrm{g} / \mathrm{día}$ o gel de testosterona al $1 \%$ (equivalente a $5 \mathrm{mg} / \mathrm{día}$ de testosterona) son los más seguros al no provocar dosis suprafisiológicas, manteniendo la efectividad, lo que ya había sido demostrado por una serie de estudios anteriores $27,28,29$.

\section{Discusión}

Los andrógenos cumplen importantes funciones en las mujeres, actuando directamente en los receptores androgénicos o a través de la aromatización a estradiol.

Desde la década de 1940 se ha intentado saber cuál es la relación entre la testosterona y la función sexual femenina; sin embargo, ha sido difícil establecerla, porque no solo depende de ella, sino también de otras hormonas y de factores biopsicosociales.

Se ha usado testosterona oral, inyectable, transdérmica y en implantes, sin lograr establecer su seguridad, eficacia y efectos adversos a largo plazo, por lo cual las principales sociedades endocrinológicas, ginecoobstétricas y de climaterio no la recomiendan, salvo para el TDSH.

En relación con los pellets, los estudios comenzaron en hombres con dosis que eran aproximadamente 10 a 20 veces mayores que las concentraciones plasmáticas de testosterona endógena de la mujer premenopáusica. Luego se iniciaron los estudios en cohortes femeninas. Existen pocos estudios aleatorizados y doble ciego de testosterona sola contra placebo. Si bien logran mejorar la función sexual en general, los niveles de testosterona son suprafisiológicos (cuatro veces 
más que los valores de testosterona endógena), por lo cual la recomendación de su uso es discutida.

Los metaanálisis realizados llegan a la conclusión de que muchos de los estudios son de casos y controles, se han realizado en cohortes pequeñas, excluyendo grupos de alto riesgo y reclutando pacientes de clínicas privadas según conveniencia (sesgo de selección). Se presentan inconsistencias y sesgos de metodología, publicación e impresión. Los estudios son limitados, de no más allá de 24 meses, por lo cual no hay evidencia de seguridad y eficacia a largo plazo. No hay datos suficientes que avalen su uso para mejorar el rendimiento cognitivo y el bienestar general, como tratamiento de enfermedades cardiovasculares ni para la prevención de enfermedad ósea.

\section{Conclusiones}

Los andrógenos sin duda cumplen funciones fundamentales en nuestro organismo, y es necesaria la comprensión de su biosíntesis y metabolismo para entender la utilidad de las terapias hormonales de testosterona y sus potenciales riesgos.

No se recomienda el uso de pellets de testosterona para ningún fin en la mujer, por producir concentraciones suprafisiológicas y por no existir evidencia en cuanto a eficacia, seguridad y efectos adversos a largo plazo.

\section{Financiamiento}

Los autores declaran no haber recibido financiamiento para este estudio.

\section{Conflicto de intereses}

Los autores declaran no tener ningún conflicto de intereses.

\section{Responsabilidades éticas}

Protección de personas y animales. Los autores declaran que para esta investigación no se han realizado experimentos en seres humanos ni en animales.

Confidencialidad de los datos. Los autores declaran que han seguido los protocolos de su centro de trabajo sobre la publicación de datos de pacientes.

Derecho a la privacidad y consentimiento informado. Los autores han obtenido el consentimiento informado de los pacientes y/o sujetos referidos en el artículo. Este documento obra en poder del autor de correspondencia.

\section{Bibliografía}

1. Burger H. Androgen production in women. Fertil Steril. 2002;77 (Suppl 4):S3-5.

2. Vegunta S, Kling JM, Kapoor E. Androgen therapy in women. J Womens Health. 2020;29:57-64.

3. American Psychiatric Association. Guía de consulta de los criterios diagnósticos del DSM-5". Madrid. Médica Panamericana. 2014.

4. Davis SR, Wahlin-Jacobsen S. Testosterone in women - the clinical significance. Lancet Diabetes Endocrinol. 2015;3:980-92.

5. Krapf J, Simon J. The role of testosterone in the management of hypoactive sexual desire disorder in postmenopausal women. Maturitas. 2009;63:213-9.

6. Traish AM, Feeley RJ, Guay AT. Testosterone therapy in women with gynecological and sexual disorders: a triumph of clinical endocrinology from 1938 to 2008. J Sex Med. 2009;6:334-51.

7. Bancroft J. The endocrinology of sexual arousal. J Endocrinol. 2005;186:411-27.

8. West SL, D'Aloisio AA, Agans RP, Kalsbeek WD, Borisov NN, Thorp JM. Prevalence of low sexual desire and hypoactive sexual desire disorder in a nationally representative sample of US women. Arch Intern Med. 2008;168:1441-9.

9. Davis SR, Davison SL, Donath S, Bell RJ. Circulating androgen levels and self-reported sexual function in women. JAMA. 2005;294:91-6.

10. McCullough A. A review of testosterone pellets in the treatment of hypogonadism. Curr Sex Health Rep. 2014;6:265-9.

11. Kapetanakis E, Dmowski WP, Auletta F, Scommegna A. Endocrine and clinical effects of estradiol and testosterone pellets used in long-term replacement therapy. Int J Gynaecol Obstet. 1982;20:387-99.

12. Cardozo L, Gibb DM, Tuck SM, Thom MH, Studd JW, Cooper DJ. The effects of subcutaneous hormone implants during the climacteric. Maturitas. 1984;5:177-84.

13. Burger HG, Hailes J, Menelaus M, Nelson J, Hudson B, Balazs N. The management of persistent menopausal symptoms with oestradiol testosterone implants: clinical, lipid and hormonal results. Maturitas. 1984;6:351-8.

14. Burger HG. Effect of combined implant of oestradiol and testosterone on livido in post menopausal women. BMJ. 1987;294:936-7.

15. Davis SR, McCloud P, Strauss BJ, Burger H. Testosterone enhances estradiol's effects on postmenopausal bone density and sexuality. Maturitas. 1995;21:227-36.

16. Glaser R, Wurtzbacher D, Dimitrakakis C. Efficacy of testosterone therapy delivered by pellet implant. $8^{\text {th }}$ European Congress on Menopause (EMAS). Maturitas. 2009;63 (Suppl 1):S1-136.

17. Glaser R. Beneficial effects of testosterone therapy in women measured by the validated Menopause Rating Scale (MRS). Maturitas. 2011;68:355-61.

18. Glaser R, Kalantaridou S, Dimitrakis C. Testosterone implants in women: pharmacological dosing for a physiologic effect. Maturitas. 2013;74:179-84.

19. Braunstein GD. Safety of testosterone treatment in postmenopausal women. Fertil Steril. 2007;88:1-17.

20. Braunstein GD. Management of female sexual dysfunction in postmenopausal women by testosterone administration: safety issues and controversies. J Sex Med. 2007;4:859-66.

21. Wierman M, Arlt W, Basson R, Davis $S$ et al. Androgen Therapy in Women: A Reappraisal: An Endocrine Society Clinical Practice Guideline. $\mathrm{J}$ Clin Endocrinol Metab, October 2014, 99(10):3489-510.

22. Davis SR, Wahlin-Jacobsen S. Testosterone in women - the clinical significance. Lancet Diabetes Endocrinol. 2015;3:908-92.

23. Islam R, Bell R, Green S, Page M, Davis S. Safety and efficacy of testosterone for women: a systematic review and meta-analysis of randomized controlled trial data. Lancet Endocrinol. 2019;7:754-66.

24. Somboonporn W, Bell RJ, Davis SR. Testosterone for peri and postmenopausal women. Cochrane Database sys Rev. 2010;(4):CD004509.

25. Johansen N, LindŽn Hirschberg A, Moen MH. The role of testosterone in menopausal hormone treatment. What is the evidence? Acta Obstet Gynecol Scand. 2020;99:966-9.

26. Davis SR, Baber R, Panay N, Bitzer J, Cerdas Perez S, Islam RM, et al. Global consensus position statement on the use of testosterone therapy for women. Climacteric. 2019;22:429-34.

27. Fooladi E, Bell RJ. Testosterone improves antidepressant-emergent loss of libido in women: findings from a randomized, double-blind, placebo-controlled trial. J Sex Med. 2014;11:831-9.

28. Panay N, Al-Azzawi F, Bouchard C, Davis SR, Eden J, Lodhi I, et al. Testosterone treatment of HSDD in naturally menopausal women: the ADORE study. Climacteric. 2010;13:121-31.

29. Shifren JL, Davis SR, Moreau M, Waldbaum A, Bouchard C, DeRogatis $L$, et al. Testosterone patch for the treatment of hypoactive sexual desire disorder in naturally menopausal women: results from the INTIMATE NM1 Study. Menopause. 2006;13:770-9. 